\title{
Kinesthetic aspects of mental representations in the identification of left and right hands
}

\author{
KAORU SEKIYAMA \\ Osaka City University, Osaka, Japan
}

\begin{abstract}
Kinesthetic aspects of mental representations of one's own hands were investigated. Line drawings showed a human hand in one of five versions, in which finger position and wrist rotation varied; each version occurred as a left and as a right hand, and could appear in any one of eight directions in the picture plane. The subject was required to make quick judgments of whether a left or a right hand was represented, under three conditions of head tilt (left, upright, right). Reaction time varied systematically, reflecting the time required to move one's own hand into congruence with the stimulus. Head tilt influenced the subjective reference frame of mental rotation when the degree of head tilt was $60 \mathrm{deg}$.
\end{abstract}

Suppose that you are presented line drawings of various versions of human hands viewed from different angles and that you are required in each case to determine quickly whether it is a left or a right hand. Intelligence tests commonly include this kind of task, in which patterns must be mentally rotated to make a certain judgment. When pictures of human hands are employed in such a task, there seem to be some particularly interesting problems. We have seen in classrooms that, to perform this task, most pupils try to move their own hands up and down. If they are inhibited from moving their hands, what will their strategy become? In other words, what kind of mental transformations will take the place of the actual movements of their own hands?

A substantial amount of work based on the paradigm of mental rotation (see Shepard, 1975, for a review) has established that reaction time (RT) increases monotonically with the angular difference between comparator and test. This pattern of RT has been confirmed for various stimulus shapes, such as threedimensional unfamiliar perspectives (Shepard \& Metzler, 1971), two-dimensional unfamiliar shapes (e.g., Cooper, 1975), alphanumeric characters (Cooper \& Shepard, 1973; Corballis, Zbrodoff, \& Roldan, 1976), and line drawings of human hands (Cooper \& Shepard, 1975). It has been reported that, when the direction of rotation is not specified, RT increases up to $180 \mathrm{deg}$ of clockwise departure and then symmetrically decreases up to $360 \mathrm{deg}$ (e.g., Cooper \& Shepard, 1973, 1975). It has also been re-

Part of this research (Experiment 1) was carried out at Waseda University. The author gratefully acknowledges the useful suggestions of Dr. Tatsuro Makino. She also wishes to thank Dr. Kiyoshi Hattori and Kazuo Bingushi for their kind help in constructing the stimuli. Requests for reprints should be sent to Kaoru Sekiyama, Department of Psychology, Osaka City University, Sumiyoshi-ku, Osaka 558, Japan. ported that, when the experimenter designates the direction of rotation in advance, the increasing function extends to 300 deg of clockwise or counterclockwise departure, depending on prior instructions (Cooper, 1975, Experiment 2).

These investigators, who support the view that mental representations are analogous to perceptions, have stressed the visual component of mental representations. It should be noted, however, that in some tasks, such as the identification of left and right hands, a kinesthetic component might be represented in the internal process. Introspection suggests that this determination is made through a kind of internalized movement of one's own hand. Cooper and Shepard (1975) used this kind of task, and, indeed, the verbal reports of their subjects suggested that their judgments were generally made by mental transformations of the visual-kinesthetic image of their own hands. Marmor and Zaback (1976) suggested mental rotation by the blind, employing same-different judgments of pairs of tactually presented forms. They accordingly argued that visual imagery is not a necessary component of mental rotation. It may well be that images preserve multimodal aspects of experience. However, Cooper and Shepard (1975) did not report any results that could not be interpreted in terms of simple visual images.

The experiments reported below investigated the multimodality of images, looking in particular for kinesthetic aspects. Cooper and Shepard used only 4 versions of the hand, palm and back of open right and left hands, depicted rather schematically. The present study used 10 versions, right and left hands in five transformational forms, depicted by line drawings.

We also examined a second problem, that of the subjective reference frame of mental rotation. Corballis and his colleagues have asked whether the subjective reference frame of mental rotation is 
defined according to the subject's retinal coordinates or gravitational ones. They carried out several experiments in which subjects faced a kind of mental rotation task with their heads upright or tilted. If the RT function shifts in proportion to head tilt, this indicates that the subjective reference frame lies closer to the retinal than to the gravitational vertical. Indeed, such a shift in RT function occurred when dot patterns were employed (Corballis \& Roldan, 1975; Corballis et al., 1976, Experiment 1). Yet the RT function was unaffected by head tilt in the case of alphanumeric characters (Corballis et al., 1976, Experiment 2). In a further investigation of the factors that determine the subjective reference frame of mental rotation, Corballis, Nagourney, Shetzer, and Stefanatos (1978) suggested that the critical factor might be the nature of the task, but their data were not conclusive. In the present experiments, we observed the effect of head tilt upon the RT function for the identification of left and right hands. Our expectation was that the subjective reference frame would be more closely related to the gravitational vertical than to the retinal one if the task included internalized movements.

\section{EXPERIMENT 1}

\section{Method}

Subjects. The subjects were 15 undergraduates at Waseda University, eight males and seven females. All were right-handed and had normal or corrected vision.

Stimuli. There were 80 stimuli obtained by rotation and reversal of five human hands; they are depicted in Figure 1. On a given experimental trial, any one of these five forms (right hands) or their mirror images (left hands) appeared in any one of eight different directions in 45-deg steps from 0 deg (upright, as illustrated in Figure 1) to $315 \mathrm{deg}$. The eight directions were defined by the clockwise angular departure from the upright.

Procedure. The subject sat at a table facing a translucent screen. Each stimulus was presented on a slide rear-projected onto the screen. The order of presentation was randomized. The subject was required to determine, as exactly and as quickly as possible, whether each stimulus was a left hand or a right hand. The index finger of each hand rested on a response button; he/she was to press the left-hand button if the stimulus represented a left hand and the right-hand button if a right hand. Subject's hands were covered with a cloth in order to prevent them from comparing their own hands with the stimuli directly. The room was illuminated. RT was measured from the onset of the stimulus to the depression of one of the response buttons. Each stimulus remained illuminated until the subject had made a response. The interstimulus interval was about $3 \mathrm{sec}$. Each stimulus subtended about 11 deg of visual angle.

Each subject was first given about 30 practice trials. Then he/she was given three sequences of trials, one with the head tilted

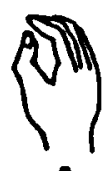

A
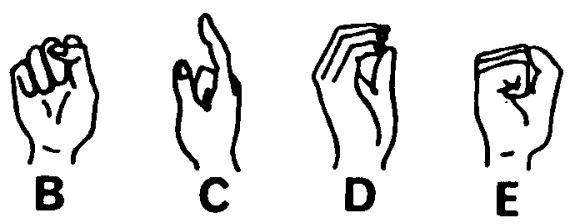

Figure 1. The five stimulus forms.
$45 \mathrm{deg}$ to the left, one with the head upright, and one with the head tilted 45 deg to the right. In the two head-tilted conditions, the subject gradually tilted his/her head until the experimenter said "stop," and then held the head at that position according to instructions. The three conditions of head tilt were counterbalanced over subjects, according to a Latin square. Each condition involved 80 trials. Rest pauses of $\mathbf{2}$ min were given between each sequence.

\section{Results and Discussion}

Reaction time as a function of angular departure. The relation between RT and angular departure of the stimulus from the upright is shown by RTs under the head-upright condition. Mean RTs, for correct responses only, were computed for each form, for left and right hand, and for each angular departure. These RTs and error rates are shown in Figure 2, as a function of angular departure. Figure 2 clearly shows that there is not a negative correlation between RT and error rate; therefore, these RTs cannot be explained by a speed-accuracy tradeoff. Average error rate was $5.1 \%$.

The RT function obtained by Cooper and Shepard (1975, Figure 4) was symmetrical about $180 \mathrm{deg}$. Their data for different hand versions were averaged, which presupposes that the shapes of the functions do not depend on stimulus versions. Indeed, Cooper (1975) found that RT functions were uniform for diverse random two-dimensional shapes.

But Figure 2 shows rather different results. First, the shape of the RT function is not always symmetrical about $180 \mathrm{deg}$. Moreover, the relation between the function for left hand and that for right hand is mirror-reversed. This is clear-cut in the case of Forms $B$ and $C$. That is, the peak of the function for these forms is at $225 \mathrm{deg}$ for left hands and at $135 \mathrm{deg}$ for right hands. Second, RT functions are not uniform for different forms. The five stimulus forms could be classified into two groups on the basis of the shape of the function, that is, A-B-C and D-E.

A three-way analysis of variance ${ }^{1}$ (left vs. right hands $\times$ forms $x$ angular departures) for repeated measures was performed on the group data. The results of the analysis can be summarized as follows. (1) The main effects of hands, forms, and angular departures were all significant $[F(1,14)=8.928$, $F(4,56)=17.898, F(7,98)=23.542$, respectively, each $\mathrm{p}<.01]$. (2) One of the two-way interactions (hands $x$ angular departures) was significant $[F(7,98)=$ $7.090, p<.01]$. (3) The three-way interaction (hands $\times$ forms $\times$ angular departures) was significant $[F(28,392)=3.232, p<.01]$. These interactions represent the mirror-reversed relation of functions between left and right hands. Although Figure 2 suggests that the trend of the RT function depended on the stimulus forms, the analysis of variance failed to reveal a forms $x$ angular departures interaction.

With respect to the effect of the stimulus forms, there was a significant difference between each pair 


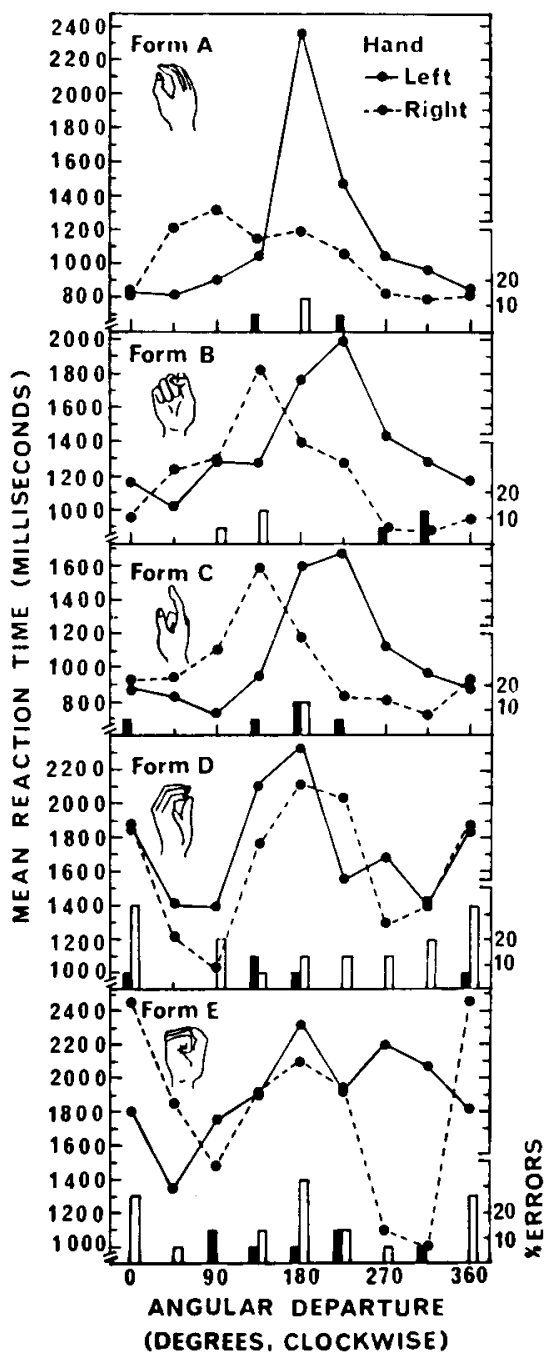

Figure 2. Mean RT as a function of angular departure of the stimulus from the upright, for the head-upright condition in Experiment 1. Error rates are plotted with solid bars representing left-hand errors and open bars representing right-hand errors.

of stimulus forms (Tukey's HSD test, $p<.01$ ) except for Forms $A$ and $E$.

Verbal reports of many subjects suggested that they transformed some "internal hand" of their own until its form and direction became identical to those of the visual stimulus. It would be natural to assume that the subject's judgments were based on a mental analogue of the actual movements of their own hands. Such a mental analogue would preserve kinesthetic or proprioceptive information attending the movements. This is inferred from (1) the mirrorreversed relation of functions between left and right hands, and (2) the similarity of functions among Forms A, B, and C, and between Forms D and E (not substantiated by the analysis of variance, however).

Consider first the mirror-reversed relation. Suppose you copy Form B at the upright position using your right hand, and rotate it in the frontal plane. If you try to rotate it clockwise, you will find that your right hand and right arm are not designed for this task. You will not be able to rotate them more than $90 \mathrm{deg}$. On the other hand, if you rotate them counterclockwise, you will be able to move them over $270 \mathrm{deg}$. Therefore, to copy Form B at the angular departure of $135 \mathrm{deg}$ (measured clockwise), you will have to rotate your right hand counterclockwise $225 \mathrm{deg}$. This predicts the longest RT to be at $135 \mathrm{deg}$. If you use your left hand, the manageable direction of rotation is the opposite. This time, RT will be the longest at $225 \mathrm{deg}$. Thus, obtained RT functions correspond to such "manageable directions" in actual movements.

This idea, that "manageable direction" in actual movements are preserved in mental transformations, can also account for similarities between forms. We have already classified the five stimulus forms into two groups (A-B-C, D-E) by the trend of RT functions. Forms $\mathrm{D}$ and $\mathrm{E}$ share a common "manageable direction" because the spatial orientation of these two is identical. They differ only in whether the hand is open or closed. Similarly, Forms A, B, and C appear to have a uniform "manageable direction" of rotation. In the case of the right hand, this seems to be counterclockwise, although the extent of possible rotation may not be equal. Of the three forms, Form A seems to have the smallest extent of possible rotation in this direction. Thus, the similarity in trend of the RT functions can be attributed to the similarity in "manageable direction" in actual movements of hands.

Effect of head tilt. The next problem to be considered is that of the subjective reference frame of mental rotation. When RT functions under different conditions of head tilt are compared, the results are as illustrated in Figure 3. At first glance, it seems that RT functions were unaffected by head tilt.

A four-way analysis of variance (head tilt $x$ forms $\times$ left vs. right hands $\times$ angular departures) for repeated measures, however, found the head tilt $X$ angular departures interaction to be significant $[F(14,196)=2.051, p<.01]$. It is not clear whether or not this interaction implies the shift of the subjective reference frame of mental rotation. As for the peak of the functions, no obvious systematic change with the head tilt is seen in Figure 3. Hence, separate analyses of variance (head tilt $\times$ left vs. right hands $x$ angular departures) were performed on RTs for each stimulus form. All but one of these analyses found the head tilt $x$ angular departures interaction to be insignificant. Only the analysis of Form A found it to be significant $[F(14,196)=2.059, p<$ $.05]$. From a visual inspection of Figure 3, this significant interaction may be attributed to the irregular fluctuation of the functions, not to the shift of the peak of the function. 


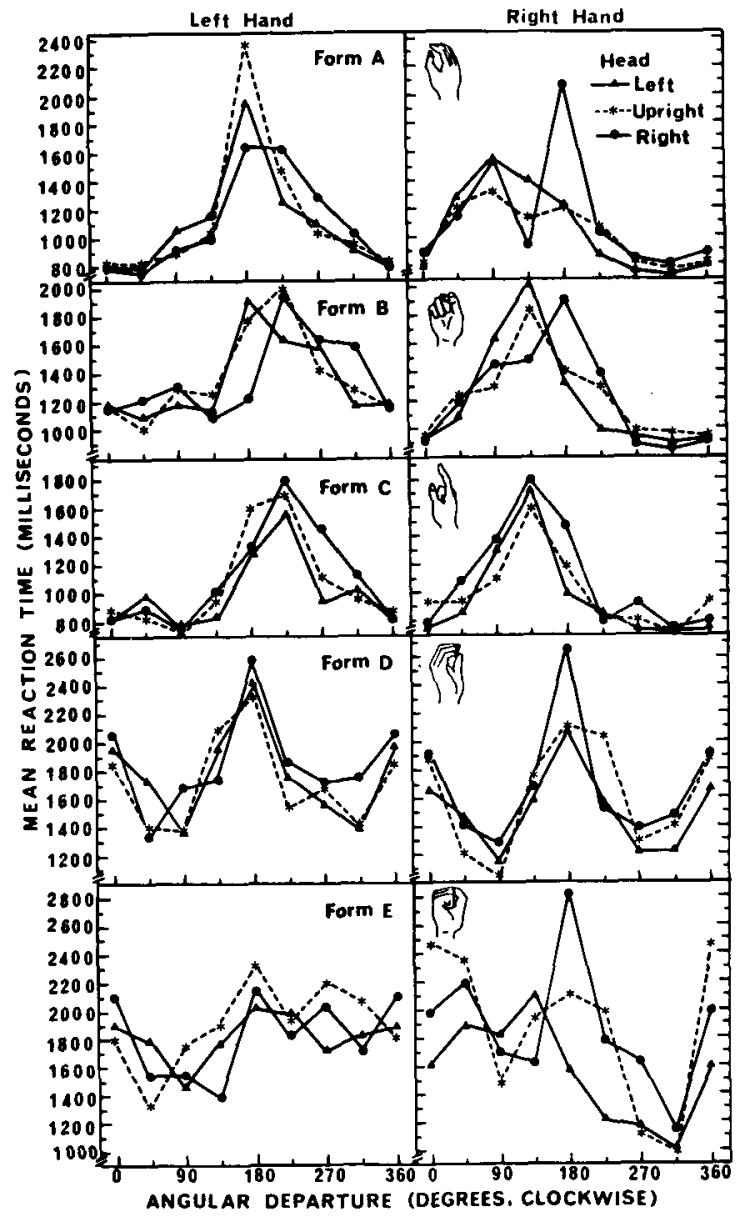

Figure 3. Mean RT as a function of angular departure, for each condition of head tilt in Experiment 1.

These results suggest that the subjective reference frame of mental rotation was aligned with the gravitational coordinates. This accords with our initial expectation, that is, that internalized movements of hands should be closely related to the gravitational coordinates. However, any firm conclusion requires experiments.

In any event, we should point out that the internal process accompanying the task might be completely different for our subjects and those of Corballis. According to our analysis, our subjects are assumed to have rotated representations of their own hands, not of the externally presented stimulus, from their canonical positions to the position of the stimulus. On the other hand, throughout his experiments (Corballis, Nagourney, et al., 1978; Corballis et al., 1976), Corballis's subjects were assumed to rotate the visual image of the externally presented stimulus from the position of the stimulus to the canonical upright position. This crucial difference may prevent the interpretation of our and their results within the same framework.

\section{EXPERIMENT 2}

The main purpose of this experiment was to test the reproducibility of the data obtained in Experiment 1. The principal modification was that the number of trials was increased. In addition, (1) the degree of head tilt was enhanced, (2) visual field was restricted by a circular frame in order to minimize visual cues about horizontal and vertical, and (3) detailed instructions were given. Modifications 1 and 2 were introduced to make the procedure identical to that of Corballis, Nagourney, et al., (1978).

\section{Method}

Subjects. The subjects were undergraduates at Osaka City University, four males and four females. All were right-handed and had normal or corrected vision.

Procedure. The procedure was essentially the same as that of Experiment 1. This time, the degree of head tilt was not $45 \mathrm{deg}$, but $60 \mathrm{deg}$, which was assured by fixing the subject's head in a helmet. The subject was given instructions to imagine "the feeling of your own hands" and to rotate "the feeling" to the position of the stimulus. The visual field was restricted by setting a pipe $(25 \mathrm{~cm}$ in diameter) between the subject's face and the screen. Each subject participated for 3 days. On each day, he/she repeated the procedure of Experiment 1 twice. In all, each subject in the present experiment experienced six times as many trials as the subjects in Experiment 1. All error trials were retaken at the end of the sequence in which they occurred.

\section{Results and Discussion}

Reaction time as a function of angular departure. Figure 4 shows RTs as a function of angular departure in the head-upright condition. Error rates are also depicted, and clearly show no speed-accuracy tradeoff. The average error rate was $4.3 \%$.

With the exception of Form A, Experiment 2 produced results similar to those of Experiment 1. We can again see in these functions the mirror-reversed relation between left and right hand. The RT functions in Figure 4, however, show smoother inflections and gentler slopes than those in Figure 2.

A three-way analysis of variance (left vs. right hands $\times$ forms $\times$ angular departures) for repeated measures was performed on the group data. The results of the analysis can be summarized as follows: (1) The main effects of hands, forms, and angular departures were all significant $[F(1,7)=15.613$, $F(4,28)=20.514, F(7,49)=10.453$, respectively, each $\mathrm{p}<.01$ ]; (2) the hands $x$ forms interaction and the hands $x$ forms $x$ angular departures interaction were significant $[F(4,28)=3.957, p<.05$, and $F(28,196)=3.543, p<.01$, respectively]. However, the analysis of variance failed to reveal a hands $x$ angular departures interaction and a forms $x$ angular departures interaction.

We can conclude that the pattern of RT function for each form was, for the most part, reproducible, although fluctuation of the functions decreased due to the increased number of trials. 


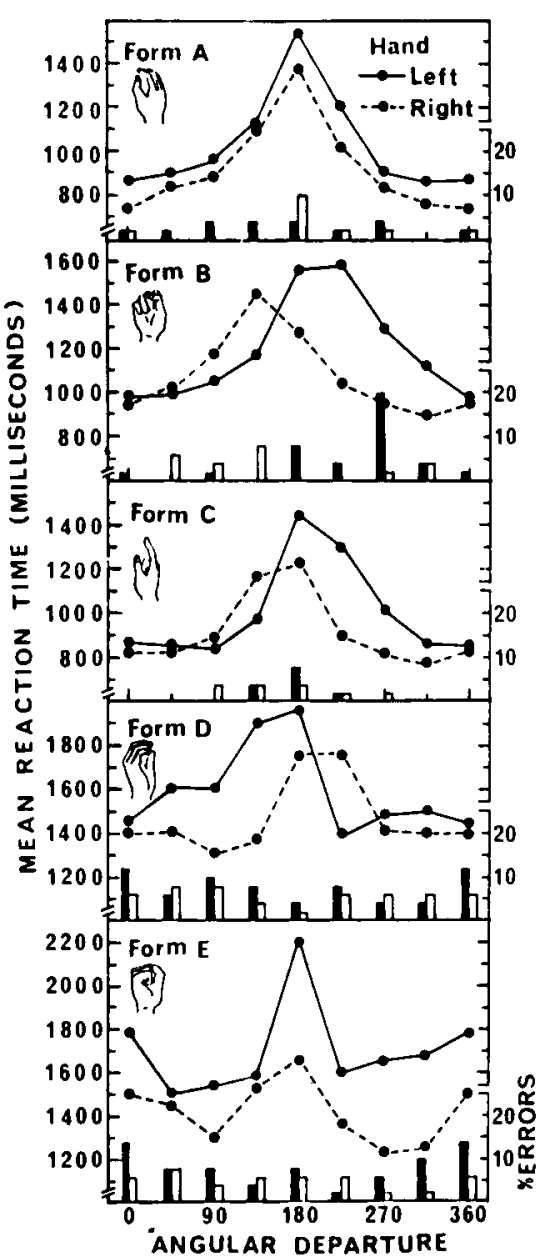

(DEGREES. CLOCKWISE)

Figure 4. Mean RT and error rate as a function of angular departure, for the head-upright condition in Experiment 2.

Individual differences. Figure 5 shows data for individual subjects under the head-upright condition for Form B. The left-hand panels are the individual functions for the left hand, the centered panels are those for the right hand, and the right-hand panels are the group data for both hands. The eight individual functions were divided into two groups on the basis of qualitative differences. The functions that have the peak at $180 \mathrm{deg}$ have been put into a $\mathrm{v}$ group. We presupposed that this shape derived from stronger effects of the visual component of internal processes. The others have been put into a $\mathrm{k}$ group, since we speculated that some kinesthetic factor might make the RT function fail to have its peak at $180 \mathrm{deg}$. The upper panels are the data for the $k$ group, and the lower panels are those for the $v$ group. This classification of individual functions seems to clarify the two aspects of the mental representation of one's own hand. If a function fails to have its peak at $180 \mathrm{deg}$, the position of its actual peak is subject to a certain rule: In this case, it was
$225 \mathrm{deg}$ for the left hand and $135 \mathrm{deg}$ for the right hand. And this rule coincides with the explanation in terms of the "manageable direction" of one's own hand, as we have discussed.

If the predominance of vision or kinesthesis depends on some individual factor, that factor is not stable. Whether a subject belongs to the $\mathbf{k}$ group or the $v$ group is variable. For instance, Subject G.K. belongs to the $\mathrm{k}$ group in the case of the right hand, but to the $v$ group in the case of the left hand.

Effect of head tilt. All the functions involving the three conditions of head tilt are shown in Figure 6. In contrast with Experiment 1, it seems that RT functions were affected by head tilt. As the head was tilted, the peak of the functions shifted in the direction of head tilt.

Analysis of variance confirmed this shift of the peak. A four-way analysis of variance (head tilt $x$ left. vs. right hands $\times$ forms $\times$ angular departures) for repeated measures found the head tilt $x$ angular departures interaction to be highly significant [F $(14,98)=6.330, p<.001]$. This interaction was then tested separately on the data for each form. According to three-way analyses of variance (head tilt $x$ left vs. right hands $\times$ angular departures), the interaction was significant for three-B, C, and Dof the five forms $[F(14,98)=2.486, F(14,98)=3.589$, and $F(14,98)=10.422$, respectively, each $p<.01]$.

These results suggest that the subjective reference frame of mental rotation was not coincident with the gravitational coordinates. This contrast between Experiments 1 and 2 could be ascribed to the degree of head tilt and/or the visual cues as to the location of the gravitational vertical.

Although we adopted much the same procedure as Corballis, Nagourney, et al. (1978, Experiment 1), the results were not identical. In their experiment, distinctive shifts of the peak of the function did not occur with head tilt. The only difference in method was the nature of the stimuli, which were alphanumeric characters in theirs and drawings of human hands in ours. Therefore, the discrepancy might arise from the stability of the canonical position of mental representations. We have learned and used characters at their canonical environmental upright position, while hands have been moved and viewed from diverse angles. Accordingly, the environmental upright position may be a more stable criterion for the mental representations of characters than for those of hands.

\section{GENERAL DISCUSSION}

This study is only a beginning in the search for evidence of kinesthetic aspects of mental representations. But significant novel features of the present experiments provide a glimpse of such kinesthetic aspects: The RT functions for left and right hands 


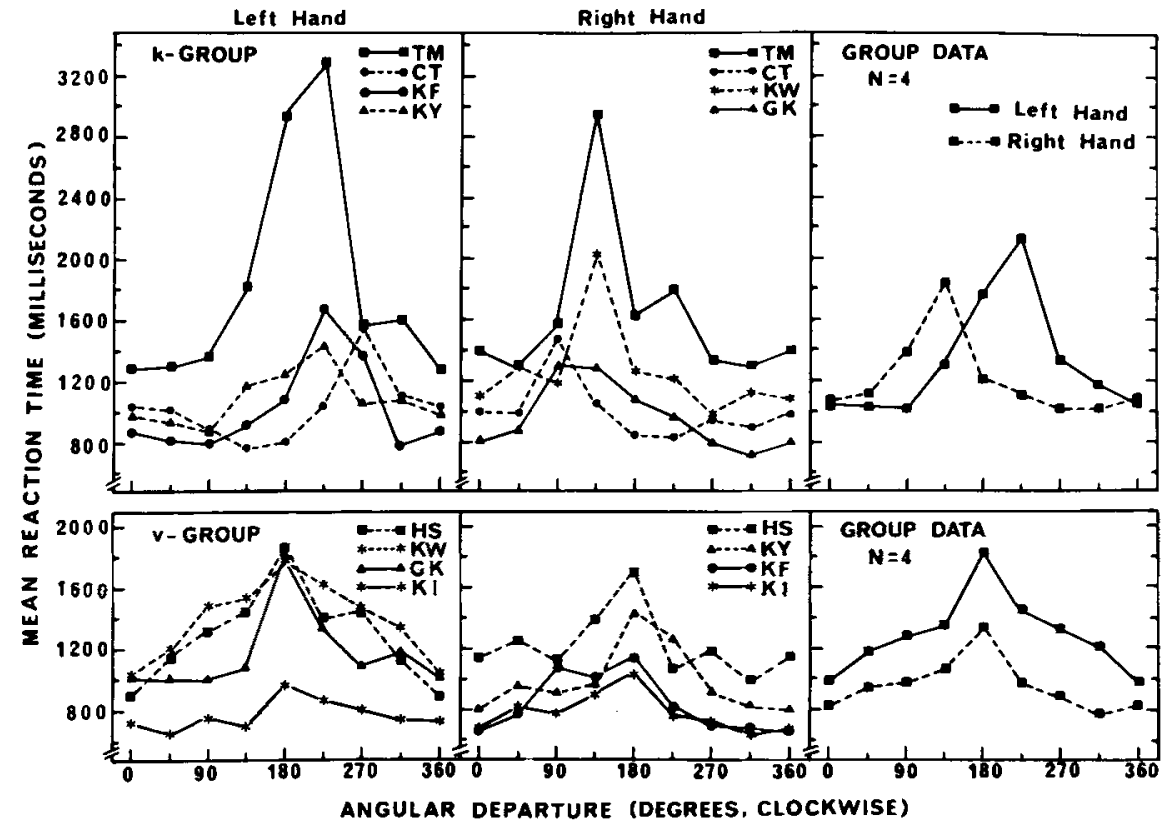

Figure 5. Mean RT as a function of angular departure for individual and group data in Experiment 2, in the case of Form $B$ under the head-upright condition.

show a systematic mirror-reversal, and the functions seem to represent "manageable directions" in actual movements of hands. It seems reasonable to assume that the mental representation of one's own hand is a visual-kinesthetic image, and that mental transformations of such images are restricted by proprioceptive information.

The present results manifest not only the kinesthetic aspects of mental representations, but also a characteristic of mental rotation. Our subjects seemed to "know" whether the presented stimulus was a left or a right hand before mental rotation. Although it may sound strange, we cannot account for the mirror-reversed relation of functions between left and right hand unless we assume two different processes for left and right hand. Indeed, some of the subjects claimed that they had initial impression about the left-right version of the stimulus, without knowing why. Similarly, in experiments using the task of identifying alphanumeric characters, Corballis, Zbrodoff, Shetzer, and Butler (1978) claimed that subjects extract at least some information concerning the version (normal vs. backward) of a stimulus prior to mental rotation. The role of mental rotation may have to do with checking or confirming a hypothesis, rather than with making the required discrimination in the first place.

We propose a tentative model of the internal process of comparing the visually presented hand and the mental representation of one's own hand. As soon as the stimulus is presented, the subject formulates a hypothesis about the left-right version of the stimulus. Then he/she generates the mental representation of either left or right hand according to the hypothesis. This representation is a visualkinesthetic image. It is first generated at its canonical position and is then moved into congruence with the stimulus. The direction of the internalized movement

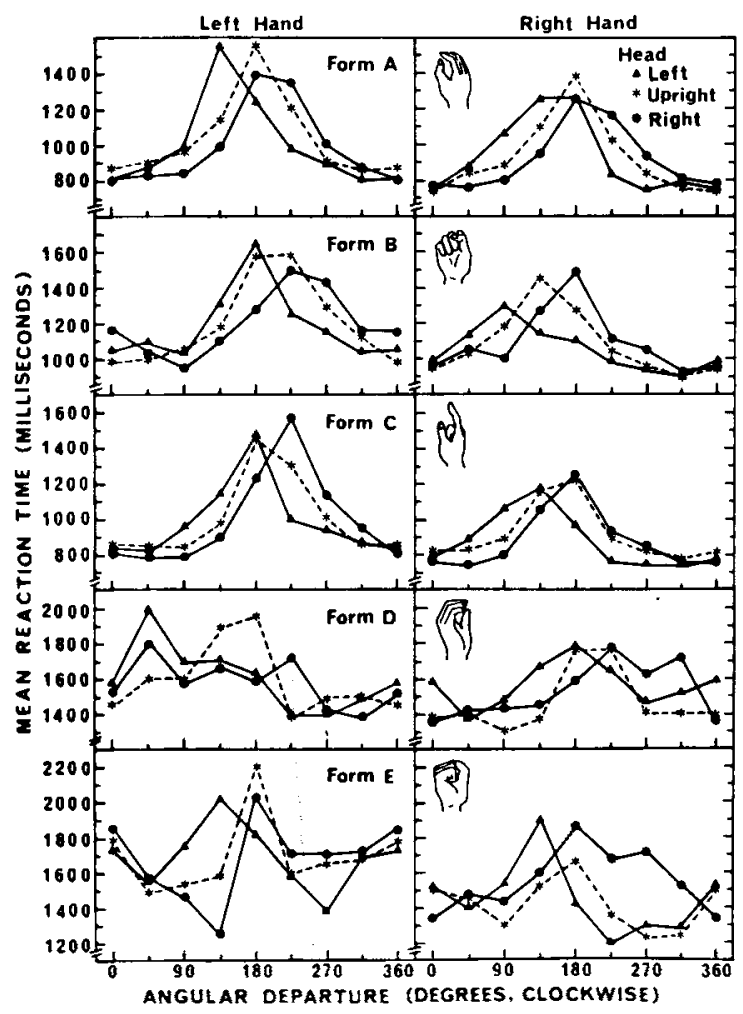

Figure 6. Mean RT as a function of angular departure, for each condition of head tilt in Experiment 2. 
is restrained by the manageable direction of the actual movement of the subject's own hand. If the image and the stimulus match, the subject makes a response that agrees with his/her first hypothesis. If they prove to be a mismatch, a response opposite to the hypothesis is made. The subject's initial hypotheses must have been correct in most cases; otherwise, the RT functions would not exhibit such a systematic relation of functions between left and right hands.

The right hand's image may be generated more easily than the left hand's, since judgments for right hands were faster than those for left hands. It is, however, not possible to partial out the contributions to this difference of the two confounded factors -left- vs. right-hand version of test stimulus and preferred vs. nonpreferred response hand.

As for the subjective reference frame of mental rotation, the outcome was not clear. At least, the results of Experiment 2 suggest that, for transformations of visual-kinesthetic images, the subjective reference frame was not coincident with the gravitational coordinates. However, this discrepancy may occur, not only because of a retinal factor, but also because of a proprioceptive factor which involved proprioceptive information originating from the subject's head tilt. The cooperation of such two factors may make the internal motor space tilted.

Finally, it is conceivable that the mental representations generated in a situation such as that in our experiments might share some properties in common with what is called "phantom limb."

\section{REFERENCES}

Cooper, L. A. Mental rotation of random two-dimensional shapes. Cognitive Psychology, 1975, 7, 20-43.
Cooper, L. A., \& She Pard, R. N. Chronometric studies of the rotation of mental images. In W. G. Chase (Ed.), Visual information processing. New York: Academic Press, 1973.

Cooper, L. A., \& Shepard, R. N. Mental transformations in the identification of left and right hands. Journal of Experimental Psychology: Human Perception and Performance, 1975, 104, 48-56.

Corballis, M. C., Nagourney, B. A., Shetzer, L. I., \& Stefanatos, G. Mental rotation under head tilt: Factors influencing the location of the subjective reference frame. Perception \& Psychophysics, 1978, 24, 263-273.

Corballis, M. C., \& Roldan, C. E. Detection of symmetry as a function of angular orientation. Journal of Experimental Psychology: Human Perception and Performance, 1975, 1 , 221-230.

Corballis, M. C., Zbrodoff, J., \& Roldan, C. E. What's up in mental rotation? Perception \& Psychophysics, 1976, 19, 525-530.

Corballis, M. C., Zbrodoff, N. J., Shetzer, L. I., \& Butler, P. B. Decisions about identity and orientation of rotated letters and digits. Memory \& Cognition, 1978, 6, 98-107.

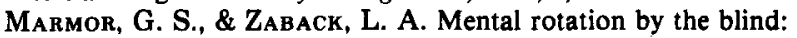
Does mental rotation depend on visual imagery? Journal of Experimental Psychology: Human Perception and Performance, 1976, 2, 515-521.

ShEPARD, R. N. Form, formation and transformation of internal representations. In R. L. Solso (Ed.), Information processing and cognition: The Loyola symposium. Hillsdale, N.J: Erlbaum, 1975.

Shepard, R. N., \& Metzler, J. Mental rotation of threedimensional objects. Science, 1971, 171, 701-703.

\section{NOTE}

1. Missing cell data in Experiment 1 were estimated from the entries of other cells within subjects, since there was only one observation in a cell. For example, when the observation was missing in a cell for $90 \mathrm{deg}$ under a condition, the estimate was obtained by averaging the data 45 and 135 deg under that condition.

(Manuscript received October 15, 1981; revision accepted for publication March 25, 1982.) 